\title{
Dissociable Performance on Scene Learning and Strategy Implementation after Lesions to Magnocellular Mediodorsal Thalamic Nucleus
}

\author{
Anna S. Mitchell, Mark G. Baxter, and David Gaffan \\ Department of Experimental Psychology, Oxford University, Oxford OX1 3UD, United Kingdom
}

\begin{abstract}
Monkeys with aspiration lesions of the magnocellular division of the mediodorsal thalamus (MDmc) are impaired in object-in-place scene learning, object recognition, and stimulus-reward association. These data have been interpreted to mean that projections from MDmc to prefrontal cortex are required to sustain normal prefrontal function in a variety of task settings. In the present study, we investigated the extent to which bilateral neurotoxic lesions of the MDmc impair a preoperatively learnt strategy implementation task that is impaired by a crossed lesion technique that disconnects the frontal cortex in one hemisphere from the contralateral inferotemporal cortex. Postoperative memory impairments were also examined using the object-in-place scene memory task. Monkeys learnt both strategy implementation and scene memory tasks separately to a stable level preoperatively. Bilateral neurotoxic lesions of the MDmc, produced by $10 \times 1 \mu \mathrm{l}$ injections of a mixture of ibotenate and NMDA did not affect performance in the strategy implementation task. However, new learning of object-in-place scene memory was substantially impaired. These results provide new evidence about the role of the magnocellular mediodorsal thalamic nucleus in memory processing, indicating that interconnections with the prefrontal cortex are essential during new learning, but are not required when implementing a preoperatively acquired strategy task. Thus, not all functions of the prefrontal cortex require MDmc input. Instead, the involvement of MDmc in prefrontal function may be limited to situations in which new learning must occur.
\end{abstract}

Key words: mediodorsal thalamus; strategy; episodic memory; amnesia; monkey; prefrontal cortex

\section{Introduction}

Damage to the magnocellular division of mediodorsal thalamic nucleus (MDmc) can cause dense amnesia in humans (Victor et al., 1989). In monkeys, substantial memory deficits in object recognition, object-in-place scene learning, and stimulus-reward association tasks follow bilateral aspiration lesions to MDmc (Aggleton and Mishkin, 1983; Zola-Morgan and Squire, 1985; Gaffan and Murray, 1990; Gaffan and Watkins, 1991; Gaffan et al., 1993; Parker et al., 1997; Gaffan and Parker, 2000). In addition, delayed matching-to-sample object recognition memory deficits have been reported after a crossed unilateral lesion of the MDmc and contralateral perirhinal cortex (Parker and Gaffan, 1998). Because this recognition memory deficit was almost as severe as the deficit produced in the same task by a crossed unilateral lesion to prefrontal cortex (PFC) and contralateral perirhinal cortex, Parker and Gaffan (1998) argued that removal of the entire MDmc produces a widespread disruption to PFC functioning that is similar to removal of the PFC itself. The broad range of deficits after MDmc damage has led to the proposal that

Received April 23, 2007; revised Aug. 10, 2007; accepted Sept. 5, 2007.

This work was supported by the Medical Research Council (A.S.M., D.G.) and the Wellcome Trust (M.G.B.). We thank M. Brown, G. Daubney, D. Kyriazis, K. Murphy, and S. Mygdal for technical support.

Correspondence should be addressed to Dr. Anna S. Mitchell, Department of Experimental Psychology, Oxford University, South Parks Road, 0xford, 0X1 3UD, United Kingdom. E-mail: anna.mitchell@psy.ox.ac.uk. D0I:10.1523/JNEUROSCI.1835-07.2007

Copyright $\odot 2007$ Society for Neuroscience $\quad$ 0270-6474/07/2711888-08\$15.00/0 the MDmc plays a general role in memory through disruption of normal functioning in the PFC (Gaffan and Parker, 2000). This proposal is further supported by the substantial reciprocal neural connections between MDmc and the PFC, especially with orbital and ventromedial regions, which are also innervated by medial temporal lobe structures including the hippocampus, amygdala, and rhinal and inferotemporal (IT) cortices (Goldman-Rakic and Porrino, 1985; Russchen et al., 1987; Ungerleider et al., 1989).

Yet an outstanding question remains regarding the extent to which damage in the MDmc affects other cognitive tasks that depend on interactions between the PFC and the medial temporal lobes, such as strategy implementation (Gaffan et al., 2002). The strategy implementation task requires monkeys to associate two different categories of objects with two different strategies for obtaining rewards. Thus efficient performance requires switching responding between the categories of objects as well as inhibiting responding to the category of objects that are not currently part of the active strategy.

The present experiment was a further test of Parker and Gaffan's (2000) proposal. We trained monkeys preoperatively on the strategy implementation task and after bilateral lesions to the MDmc assessed impairments in implementation of this conditional strategy. The secondary aim of the present study was to develop successful neurotoxic lesions within the MDmc in the macaque. Previous lesion techniques have involved aspiration of the MDmc. Although this technique produces reliable lesions of 
MDmc as well as memory impairments, it may have caused unintended damage to fibers of passage coursing through the medial thalamus, thus adding to the amnesic deficits. To gauge the success of our neurotoxic lesions we compared the effects of our current lesions with those of MDmc ablations from a previous study (Gaffan and Parker, 2000) in scene learning, a rapid new learning memory task that is in some ways characteristic of episodic memory in humans (Gaffan, 1994).

\section{Materials and Methods}

Subjects. Subjects were seven rhesus monkeys (Macaca mulatta), four unoperated controls (CON1-CON4) and three that received bilateral neurotoxic lesions of MDmc (MD1-MD3), six males (CON1, CON2, CON4, MD1, MD2, and MD3) and one female (CON3), 3.59-7.44 kg (28 -51 months old) at the start of behavioral training. Two monkeys (CON1 and CON2) had learned several visual discrimination problems on a touch-screen apparatus before entering the present study. These animals began training on the strategy implementation task and then acquired the scene memory task. The other five monkeys (CON3, CON4, MD1, MD2, and MD3) underwent pretraining, and then learned the scene memory task followed by the strategy implementation task. Each monkey was preoperatively trained to a stable level of performance before surgery. The preoperative and postoperative performance tests were identical for all seven monkeys. All experimental procedures were performed in compliance with the United Kingdom Animals (Scientific Procedures) Act of 1986.

Apparatus. All behavioral testing took place in an automated apparatus. Each monkey was taken from the home enclosure into the test cubicle in a wheeled transport cage, which was fixed in front of a video-display unit with a touch-sensitive screen $(380 \times 280 \mathrm{~mm}, 800 \times 600$ pixel resolution). The monkey could reach through horizontally oriented bars ( $\sim 45 \mathrm{~mm}$ apart) at the front of the cage to reach the screen and the rewards. Stimulus presentation, recording of touches to the screen, and reward delivery were all under computer control. A pellet dispenser delivered $190 \mathrm{mg}$ banana-flavored or sugar pellets (P. J. Noyes, Lancaster, $\mathrm{NH}$ ) into a hopper located centrally underneath the touch screen. Pellet delivery produced a click from the pellet dispenser as well as a $500 \mathrm{~ms}$ tone from the computer. A metal "lunchbox" $(\sim 200 \times 100 \times 100 \mathrm{~mm})$ was located to the left of the hopper and was filled with a mixture of wet monkey chow, seeds, apple, banana, orange, nuts, and dates. Infrared cameras positioned at different locations within the test cubicle allowed the experimenter in another room to watch the monkey. Testing was conducted in the dark except for the illumination from the video screen.

Behavioral testing: pretraining. The two monkeys that had experience with a discrimination learning task using the touch screen apparatus (Baxter and Gaffan, 2007) had no additional pretraining before beginning training in the strategy implementation task (described below). The remaining monkeys were shaped to enter a transport cage from their home enclosure, and once they were reliably eating food while positioned at the apparatus in the test cubicle, pretraining began. Animals were initially trained to touch objects on the touch screen by autoshaping procedures. First, reward pellets were delivered on a variable-interval (2 min) schedule to accustom them to take pellets in the test cubicle. After several days of pellet training, the touch screen was activated and the screen was filled with an array of different-colored alphanumeric characters on a black background (in a different size and typeface than those used in the main task). Touches to any location on the screen resulted in pellet delivery. In the third stage, single alphanumeric characters were presented in random locations on the screen, and remained until touched; a touch caused the character to disappear and a reward pellet to be delivered. Gradually, the complexity of the display was increased by introducing additional visual elements (a colored background, colored ellipse segments, and a single large alphanumeric character). When monkeys were reliably completing 50 trials in a single test session with minimal accuracy errors (i.e., touching any location on the screen other than the small alphanumeric character), they were ready for training on the scene memory task. The monkeys with discrimination learning experi- ence underwent this third stage of pretraining between acquisition of the strategy task and the scene task.

Scene memory task. The object-in-place scene memory task was adapted from Gaffan (1994). The stimulus material was identical to those described in detail by Gaffan (1994) and Browning et al. (2005). Example stimuli of the scenes are displayed in the supplemental material (available at www.jneurosci.org). Briefly, each trial consisted of an artificially constructed scene, which occupied the whole area of the display screen. Foreground objects, of which there were two in each scene, consisted of randomly selected small colored typographic characters each placed in a constant location within the individual scene. One of these two foreground objects was the correct (rewarded) one for the monkey to touch and the other was incorrect (nonrewarded). The background of each scene was generated using an algorithm based on a random number generator. Each scene was unique in that they varied in several randomly selected attributes including (1) the background color of the screen, (2) the location of ellipses on the screen, (3) the color, size and orientation of ellipse segments, (4) the typographic character, clearly distinct in size from the foreground objects, and (5) the color of the typographic character. All the colors were assigned with the constraint that the foreground objects should be visible (that is, there was a minimum separation in color space between the colors of a foreground object and the color of any element of its local background). Because these scenes were randomly generated, an infinite number of unique scenes could be presented.

After each monkey learned to touch single foreground objects against a black background (as described in the pretraining section above), additional scene elements were introduced in shaping programs until the monkey reliably touched the foreground object when presented with a new scene. Problems were then introduced with two foreground objects (one correct and one incorrect, as described above) and the number of scenes given in each session was gradually increased, based on each monkey's performance.

In the final version of the task, within-session learning involved presenting 20 new scenes in each session that were repeated eight times. Each scene was presented once in each of the eight repetitions per session using the same order of presentation of the 20 scenes. A touch to the correct object caused the object to flash for $2 \mathrm{~s}$, then the screen blanked and a reward pellet was delivered into the hopper. A touch to the incorrect object caused the screen to blank immediately, no reward was given and an intertrial interval imposed for $10 \mathrm{~s}$. For the first repetition of the list of new scenes only, incorrect responses were followed by a correction trial in which the scene was re-presented with only the correct object present. Touches anywhere else in the scene caused the screen to blank and the trial was repeated. Monkeys learned which object in each scene was correct by trial and error, generally very rapidly during the first run through the list, because error rates were very low during the second repetition of the list (14-26.5\%; chance is 50\%). When the monkey completed the final trial of a session the lunchbox opened automatically, the monkey received the large food reward and was allowed $\sim 10$ min to eat some of the food and take the remainder into the cheek pouches before being returned to the home enclosure. If the final trial was incorrect, a correction trial was given so that the monkey only ever received the large food reward after a correct response. The dependent measure was the number of errors (initial touches of the incorrect foreground object) in each repetition block of the 20 scenes. Training continued until performance was stable. On completion of scene memory training, monkeys (MD1, $\mathrm{MD} 2$, MD3, CON3, and CON4) were trained on strategy implementation.

Strategy implementation task. This task is identical to that described by Gaffan et al. (2002) except that clip-art stimuli were used instead of compound alphanumeric characters. The strategy implementation task required monkeys to learn about two categories of objects. Each category was associated with a different strategy that had to be performed to obtain food reward. The first category of rewards ("persistent") required the monkey to choose objects from this category for four trials in a row to receive reward. The second category of rewards ("sporadic") required monkeys to choose objects from this category any time after receiving a reward from the persistent category. In the sporadic category a reward was received after a single choice of the correct object but another spo- 
Table 1. Ratio of trials to rewards in the strategy task for preoperative and postoperative retention

\begin{tabular}{lcc}
\hline Monkey & Pre & Post \\
\hline C1 & 3.1 & 3.3 \\
C2 & 3.0 & 2.9 \\
C3 & 3.0 & 3.4 \\
C4 & 3.1 & 3.3 \\
Control mean & 3.1 & 3.2 \\
MD1 & 3.1 & 3.4 \\
MD2 & 3.1 & 3.4 \\
MD3 & 3.1 & 3.5 \\
MD mean & 3.1 & 3.4 \\
\hline
\end{tabular}

Pre, Preoperative ratio; Post, postoperative ratio.

radic reward was not given until another persistent reward had been earned. The monkey had to learn by trial and error which object was from which category of reward. The same four pairs of objects were used for all monkeys throughout all preoperative and postoperative training and testing (see photos of the objects in the supplemental material, available at www.jneurosci.org.).

On each trial a pair of objects appeared on the screen, containing one object from each category, and the monkey was allowed to choose one of the two. The background for each trial was always black. After each choice both objects disappeared and an intertrial interval of $5 \mathrm{~s}$ began, during which any touch to the screen reset the interval. In each session, monkeys chose objects across trials until they had earned 50 rewards. The dependent measure was the trials/reward ratio. Thus, the choice strategy that would optimize the rate of reward delivery was for the monkey to choose the persistent object on four consecutive trials, then the sporadic object on the following trial, and then to repeat this sequence of choices resulting in two rewards for every five trials (a trials/reward ratio of 2.5). Thus, the optimal strategy requires alternating choices between persistent and sporadic objects, with the switch occurring when reward had been earned for selection of one category. This is illustrated by the sequence pppPSpppPS, where a lowercase $\mathrm{p}$ is a persistent choice, an uppercase $\mathrm{P}$ is a rewarded persistent choice, and an uppercase $\mathrm{S}$ is a rewarded sporadic choice.

Strategy implementation: behavioral testing. Training procedures were identical to Gaffan et al. (2002) and proceeded in five phases. Briefly, for each of the first four phases of training monkeys were presented with one pair of objects (one persistent, one sporadic) at a time in each session until the trials/reward ratio of 2.94 or lower was reached in each of two consecutive sessions in which 50 total rewards were earned, or until a total of 6000 (first problem) or 4000 (all other phases) rewards had been earned. After reaching this criterion with one pair of objects, the same procedure was repeated with the three additional pairs of objects (phases two through four). Once this criterion was achieved with each pair individually, phase five involved the four pairs of objects being presented in each session randomly intermixed across trials so choice behavior had to be guided by the category membership of each object rather than a sequence of specific object choices. Training in this final phase continued to the same criterion (two consecutive sessions with a trials/reward ratio of 2.94 or better or 4000 rewards earned, $\sim 80$ sessions of training). Choice behavior was above chance in the first session of the current experiment with intermixed problems (mean trials/reward ratio, 4.43); chance performance would be 16.3 (Gaffan et al., 2002). Monkeys that did not reach the 2.94 trials/reward ratio criterion and advanced based on the cumulative number of rewards earned within a phase (CON2, third problem and final phase; CON4, final phase) performed comparably in their preoperative performance test to other monkeys that had achieved the criterion during training (Tables 1,2 ). On completion of strategy implementation training, $\mathrm{CON} 1$ and $\mathrm{CON} 2$ were trained on scene learning.

Performance tests. After completion of training on both the scene learning and strategy implementation tasks, all monkeys were given an identical preoperative performance test consisting of 24 sessions. The first session was object-in-place scene memory (day 1), followed by five cycles of two sessions of strategy implementation followed by two ses-
Table 2. Ratio of trials to rewards in the strategy task for preoperative and postoperative retention

\begin{tabular}{llll}
\hline Monkey & Pre & Post 1 & Post 2 \\
\hline S1 & 3.0 & 3.4 & 8.6 \\
S2 & 2.9 & 3.1 & 6.3 \\
S3 & 2.9 & 3.1 & 9.3 \\
FC X IT mean & $\mathbf{2 . 9}$ & $\mathbf{3 . 2}$ & $\mathbf{8 . 1}$ \\
\hline
\end{tabular}

Data are presented by Gaffan et al. (2002). Pre, Preoperative ratio; Post 1, ratio after first surgery to produce unilateral lesions of either FC or IT; Post2, ratio after second surgery to produce the combined FC $\times$ IT disconnection.

sions of object-in-place scene memory (days 2-21), followed by two sessions of strategy implementation (days 22-23) then a final testing session (day 24) of scene memory. This sequence of performance testing is illustrated as SIISSIISSIISSIISSIISSIIS, where "S" is scene memory and "I" is strategy implementation.

Data from the first four sessions were not included in the behavioral analyses, leaving 20 sessions of performance data (10 of scene memory; 10 of strategy implementation). In this double-alternation design we could compare performance on each task when it was preceded by performance on the same or a different task, although we did not observe any systematic variation in performance related to this variable either before or after surgery. This test was repeated in the same way beginning at least 2 weeks after surgery (for monkeys in the MDmc lesion group) or an equivalent period of rest for the four control monkeys.

Surgery. Monkeys MD1-MD3 received bilateral neurotoxic lesions to the magnocellular division of the mediodorsal thalamic nucleus in one neurosurgical procedure. Neurosurgical procedures were performed in a dedicated operating theater under aseptic conditions. Monkeys were given steroids (MD1 and MD2, dexamethasone, $1 \mathrm{mg} / \mathrm{kg}$; MD3, methylprednisolone, $20 \mathrm{mg} / \mathrm{kg}$ ) the night before surgery (i.m.), and two (MD1 and MD2, dexamethasone, 1-2 mg/kg) or three doses (MD3, methylprednisolone, $20 \mathrm{mg} / \mathrm{kg}$ ) were given $4-6 \mathrm{~h}$ apart (i.v. or i.m.) on the day of surgery to protect against intraoperative edema and postoperative inflammation. Each monkey was sedated on the morning of surgery with both ketamine $(10 \mathrm{mg} / \mathrm{kg})$ and xylazine $(0.5 \mathrm{mg} / \mathrm{kg})$. Once sedated, the monkey was given atropine $(0.05 \mathrm{mg} / \mathrm{kg})$ to reduce secretions, antibiotic (amoxicillin, $8.75 \mathrm{mg} / \mathrm{kg}$ ) for prophylaxis of infection, opioid (buprenorphine $0.01 \mathrm{mg} / \mathrm{kg}$, i.v., repeated twice at $4-6 \mathrm{~h}$ intervals on the day of surgery, i.v. or i.m.) and nonsteroidal anti-inflammatory (meloxicam, $0.2 \mathrm{mg} / \mathrm{kg}$, i.v.) agents for analgesia, and an $\mathrm{H} 2$ receptor antagonist (ranitidine, $1 \mathrm{mg} / \mathrm{kg}$, i.v.) to protect against gastric ulceration as a side effect of the combination of steroid and nonsteroidal anti-inflammatory treatment. The head was shaved and an intravenous cannula put in place for intraoperative delivery of fluids (warmed sterile saline drip, $5 \mathrm{ml} / \mathrm{h} / \mathrm{kg}$ ). The monkey was moved into the operating theater, intubated, placed on isoflurane anesthesia (MD1 and MD2, 1.2-2.70\%, to effect, in $100 \%$ oxygen) or sevoflurane (MD3, 2.25-4.0\%, to effect, in 100\% oxygen) and then mechanically ventilated. Adjustable heating blankets allowed maintenance of normal body temperature during surgery. Heart rate, oxygen saturation of hemoglobin, mean arterial blood pressure, end tidal $\mathrm{CO}_{2}$, body temperature, and respiration rate were monitored continuously throughout surgery.

The monkey was placed in a stereotaxic head holder and the head cleaned with alternating antimicrobial scrub and alcohol and draped to allow a midline incision. The skin and underlying galea were opened in layers. The temporal muscles were retracted as necessary to expose the skull surface over the intended lesion site. A D-shaped bone flap was turned over the right hemisphere and the midline. The dura over the posterior part of the right hemisphere was cut and retracted to the midline. The right hemisphere was retracted with a brain spoon and the splenium of the corpus callosum was cut in the midline with a glass aspirator. The tela choroidea (the membrane covering the third ventricle and the thalamus) was cauterized in the midline posterior and dorsal to the thalamus using a metal aspirator that was insulated to the tip. The posterior commissure, the third ventricle posterior to the thalamus and the most posterior $5 \mathrm{~mm}$ of the midline thalamus were exposed. A stereotaxic manipulator holding a $10 \mu \mathrm{l}$ Hamilton syringe with a blunt tipped 26-gauge needle with its hole $1 \mathrm{~mm}$ above the base was positioned 
above the posterior commissure at the midline using the third ventricle as a guide. Neurotoxic bilateral lesions to the magnocellular division of the mediodorsal thalamic nucleus in subjects MD1, MD2, and MD3 were produced by $10 \times 1 \mu \mathrm{l}$ injections of a mixture of ibotenic acid $(10 \mathrm{mg} / \mathrm{ml}$; Biosearch Technologies, Novato, CA) and NMDA (10 mg/ml; Tocris, Bristol, UK) dissolved in sterile $0.1 \mathrm{~m}$ PBS. The monkey brain atlas of Ilinsky and Kultas-Ilinsky (1987) was used to calculate the intended lesion site coordinates. The needle was positioned for the first pair of coordinates: anteroposterior (AP), $+5.2 \mathrm{~mm}$ anterior to the posterior commissure; mediolateral (ML), $\pm 1.2 \mathrm{~mm}$ lateral to the third ventricle; dorsoventral (DV), $-4.0 \mathrm{~mm}$ (to compensate for the hole positioned 1 $\mathrm{mm}$ above the tip of the needle) ventral to the surface of the thalamus directly above the intended lesion site. Each injection was made slowly over $4 \mathrm{~min}$ and the needle was left in place for $\sim 4$ min before being moved to the next site. The needle was then repositioned for the second pair of coordinates: AP, $+4.2 \mathrm{~mm}$; ML, $\pm 1.5 \mathrm{~mm}$; DV,$-5.0 \mathrm{~mm}$. The third, fourth and fifth pairs of coordinates were AP, $+4.2 \mathrm{~mm}, \mathrm{ML}, \pm 1.5$ $\mathrm{mm}$, and $\mathrm{DV},-3.0 \mathrm{~mm}$; AP, $+3.4 \mathrm{~mm}, \mathrm{ML}, \pm 1.7 \mathrm{~mm}$ and $\mathrm{DV},-4.0$ $\mathrm{mm}$; and $\mathrm{AP},+3.4 \mathrm{~mm}, \mathrm{ML}, \pm 1.7 \mathrm{~mm}$ and $\mathrm{DV},-3.0 \mathrm{~mm}$, respectively. In each case, the DV coordinate was relative to the surface of the thalamus at the injection site.

When the lesions were complete, the dura was repositioned but not sewn, the bone flap was replaced and held with loose sutures, and the skin and galea were closed in layers. The monkey was removed from the head-holder and anesthesia discontinued. The monkey was extubated when a swallowing reflex was observed, returned to the home cage, and monitored continuously until normal posture was regained. Nonsteroidal anti-inflammatory analgesic (meloxicam, $0.2 \mathrm{mg} / \mathrm{kg}$, oral) and antibiotic $(8.75 \mathrm{mg} / \mathrm{kg}$, oral) treatment continued after surgery in consultation with veterinary staff, typically for $5 \mathrm{~d}$.

Histology. After completion of all behavioral testing each monkey was sedated with ketamine $(10 \mathrm{mg} / \mathrm{kg})$, deeply anesthetized with intravenous barbiturate and transcardially perfused with $0.9 \%$ saline followed by $10 \%$ formalin. The brains were cryoprotected in formalin-sucrose and then sectioned coronally on a freezing microtome at $50 \mu \mathrm{m}$ thickness. A 1 -in-10 series of sections was collected throughout the thalamus that was expanded to a 1-in-5 series through the area of the lesion; these were mounted on gelatin-coated glass microscope slides and stained with cresyl violet.

MDmc lesions. Figure 1 shows schematic diagrams depicting the posterior medial thalamus as well as photomicrographs of cresyl violet stained coronal sections corresponding as closely as possible to the schematic diagrams for the three MDmc lesioned monkeys (MD1, MD2, MD3). All three monkeys in the MDmc group had extensive bilateral lesions in the magnocellular division of the mediodorsal thalamic nucleus as intended. The largest lesion was in monkey MD1, with slight sparing in the most rostral and caudal extents of the MDmc. There was also some slight damage in the anterodorsal thalamic nucleus and the most anterior portion of the laterodorsal thalamic nucleus of the right hemisphere (Fig. 1, column 2, photos 1-3). The lesion in MD3 was as extensive as that of MD1 within the MDmc. There was also some unilateral damage in the anterior thalamic nuclei of the left hemisphere (Fig. 1, column 4, photos 1 and 2). The MDmc damage in MD2 was slightly smaller than the other two lesions, with more extensive atrophy in this lesion within the caudal aspects of the MDmc. In the more rostral parts of MDmc the dorsal area was completely spared in this monkey (see Fig. 1, column 3, photo 3). There was also some slight damage to the right posterior cingulate and right retrosplenial cortex in this monkey. Other thalamic damage outside the MDmc (excluding the left laterodorsal thalamic nucleus for MD1 and the right anteroventral thalamus for MD3) was similar in all three lesions with damage to the rhomboid, reuniens, and central medial nuclei. The paraventricular nucleus of the epithalamus was extensively damaged throughout the entire anteroposterior extent of the lesions. All three monkeys also had sagittal section of the splenium of the corpus callosum and hippocampal commissure dorsal to the posterior thalamus [note that this section does not affect performance on the scene learning task (see Parker and Gaffan, 1997)].

\section{Results}

\section{Scene memory}

The rate of new scene learning was measured as the mean number of errors made in repetition trials 2-8 in the final 10 sessions of the scene memory task during the double alternation performance test and is shown in Figures 2 and 3, and Table 3. It is clear that learning of the new lists of 20 scenes in each session was fast and proficient during the preoperative performance test.

Postoperative new learning in the scene memory task during the performance test is shown in Figures 2 and 3 and Table 3. Bilateral neurotoxic lesions to the magnocellular mediodorsal thalamic nucleus produced a severe impairment in new scene learning, with these operated monkeys committing almost five times as many errors postoperatively, compared with control monkeys whose error rates were similar before and after a period of rest equivalent in time to postoperative recovery for the operated group. A two (group, control vs MDmc) by two (testing phase, preoperative vs postoperative) repeated-measures ANOVA revealed a main effect of group $\left(F_{(1,5)}=14.48 ; p=\right.$ $0.013)$, main effect of testing phase $\left(F_{(1,5)}=11.39 ; p=0.02\right)$, and a group by testing phase interaction $\left(F_{(1,5)}=11.63 ; p=0.02\right)$.

Figure 2 and Table 3 also include preoperative and postoperative performance from two previously reported groups of monkeys who were tested in the same apparatus using the same scene memory task but with 10 consecutive sessions of training by Browning et al. (2005) and Gaffan and Parker (2000). First the preoperative data in Table 3 clearly indicates that the double alternation performance test for the current monkeys did not impair their proficiency at the task when compared with monkeys only learning this scene memory task across consecutive sessions. Furthermore the postoperative impairments in new scene memory learning are comparable, with damage to the MDmc of the current study causing similar new learning deficits to those following bilateral ablations to the MDmc (see Table 3) (Gaffan and Parker, 2000) as well as unilateral frontal cortex and contralateral IT ablations (Fig. 2) (Browning et al., 2005).

Given the extent of the new learning impairments after the MDmc lesions, we considered it necessary to assess the types of postoperative performance errors during the scene memory task. Types of response errors across the eight trials per session were subdivided based on whether the initial response during the first trial was correct (1C) or incorrect (1W). An involvement of the MDmc in behavioral flexibility could impair scene learning because monkeys continue emitting initial incorrect responses and do not change them in response to feedback. This would predict that performance would be more impaired for scenes in which the initial response was incorrect compared with scenes in which the initial response was correct. Comparison of responding between preoperative and postoperative testing phases revealed expected main effects of trial, test phase, $1 \mathrm{C} / 1 \mathrm{~W}$, and group, as well as an interaction of $1 \mathrm{C} / 1 \mathrm{~W}$ with trial, an interaction of $1 \mathrm{C} / 1 \mathrm{~W}$ with group $\left(F_{(1,5)}=18.13 ; p=0.008\right)$, a three-way interaction of $1 \mathrm{C} / 1 \mathrm{~W}$, test phase, and group $\left(F_{(1,5)}=10.29 ; p=0.03\right)$, and a four-way interaction of $1 \mathrm{C} / 1 \mathrm{~W}$, test phase, group, and trial $\left(F_{(6,30)}=2.48 ; p=0.05\right)$. These data are plotted in Figure $4 a$. These results suggest that poor performance after the bilateral neurotoxic lesions could be explained by an increased perseverative tendency to continue to respond incorrectly to scenes where the initial response is incorrect. However, as Figure $4 a$ clearly shows, the number of errors in trials after an initial correct response also increased significantly, thus, the poorer postoperative performance of the MDmc lesion group cannot be explained by 


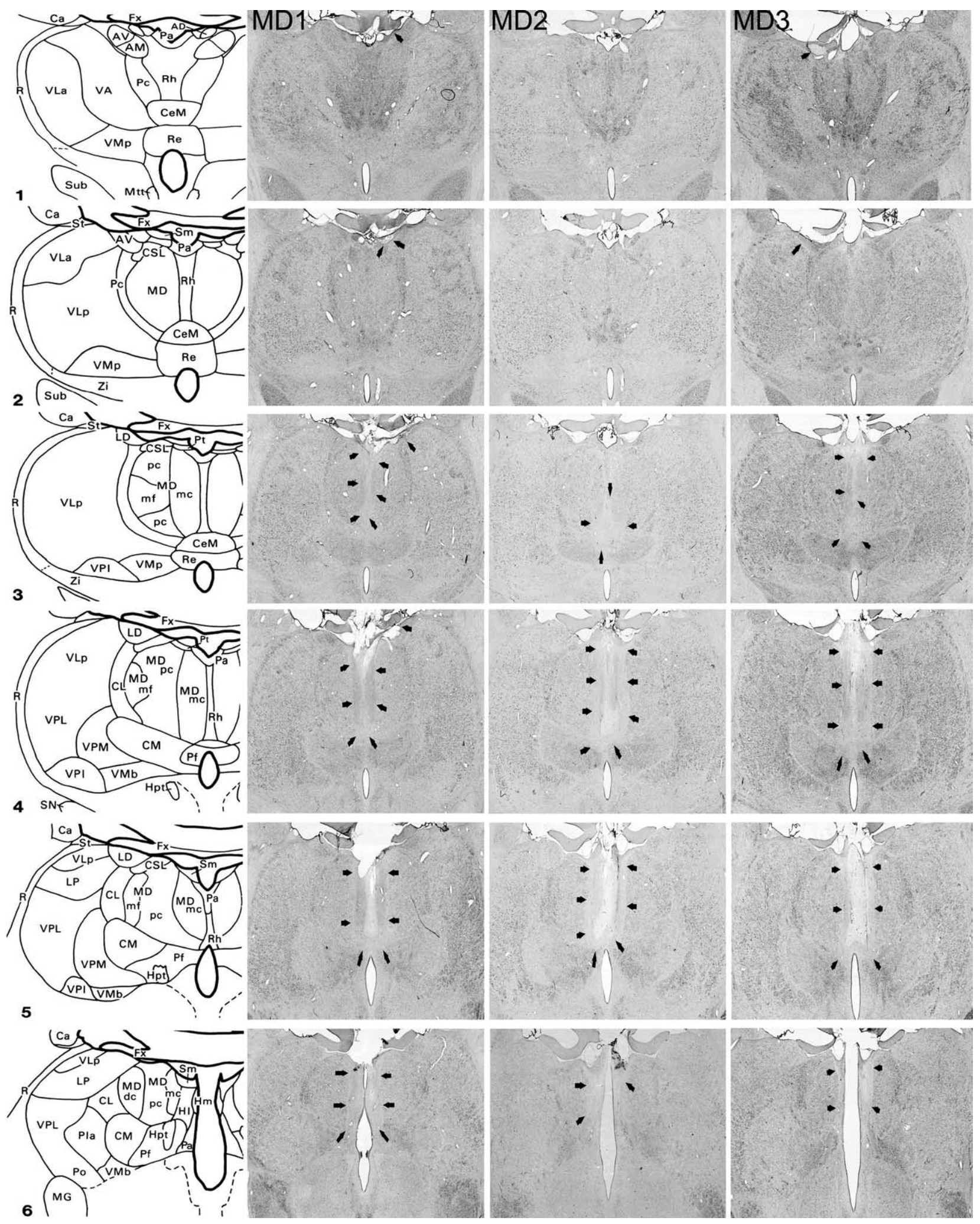

Figure 1. MDmc lesions. Schematic diagrams of six sections, $1 \mathrm{~mm}$ apart, through the posterior medial thalamus of a monkey taken from Gaffan and Murray (1990). The next three columns show photomicrographs of the MDmc lesions for MD1, MD2, and MD3 corresponding as closely as possible to the each of the schematic diagrams. Arrowheads indicate the site of each lesion. AD, Anterodorsal nucleus; $\mathrm{AM}$, anteromedial nucleus; $\mathrm{AV}$, anteroventral nucleus; $\mathrm{Ca}$, caudate nucleus; CeM, central medial nucleus; $\mathrm{CL}$, central lateral nucleus; $\mathrm{CM}$, center median nucleus; $\mathrm{CSL}$, central superior lateral nucleus; FX, fornix; HI, lateral habenular nucleus; $\mathrm{Hm}$, medial habenular nucleus; Hpt, habenulopeduncular tract; IT, inferotemporal cortex; LD, lateral dorsal nucleus; Li, limitans nucleus; LP, lateral posterior nucleus; MD, mediodorsal nucleus; MDdc, mediodorsal nucleus, densocellular portion; MDmc, magnocellular portion; MDmf, mediodorsal nucleus, multiformis portion; MDpc, mediodorsal nucleus, parvocellular portion; MG, medial geniculate complex; Mm, mamillary complex; Mtt, mamillothalamic tract; $\mathrm{Pa}$, paraventricular nucleus; $\mathrm{Pc}$, paracentral nucleus; Pf, parafascicular nucleus; Pla, anterior pulvinar nucleus; Pli, inferior pulvinar nucleus; Pl, lateral pulvinar nucleus; $\mathrm{Po}$, posterior nucleus; $\mathrm{Pa}$, parataenial nucleus; $\mathrm{R}$, reticular nucleus; Re, reuniens nucleus; Rh, rhomboid nucleus; SG, suprageniculate nucleus; SN, substantia nigra; St, stria terminalis; Sub, subthalamic nucleus; VA, ventral anterior nucleus; VLa, ventral lateral nucleus, anterior portion; $\mathrm{VLp}$, ventral lateral nucleus, posterior portion; VMb, ventral medial nucleus, basal portion; VMp, ventral medial nucleus, principal portion; VPi, ventral posterior inferior nucleus; VPI, ventral posterior lateral nucleus; VPm, ventral posterior medial nucleus; Zi, zona incerta. 


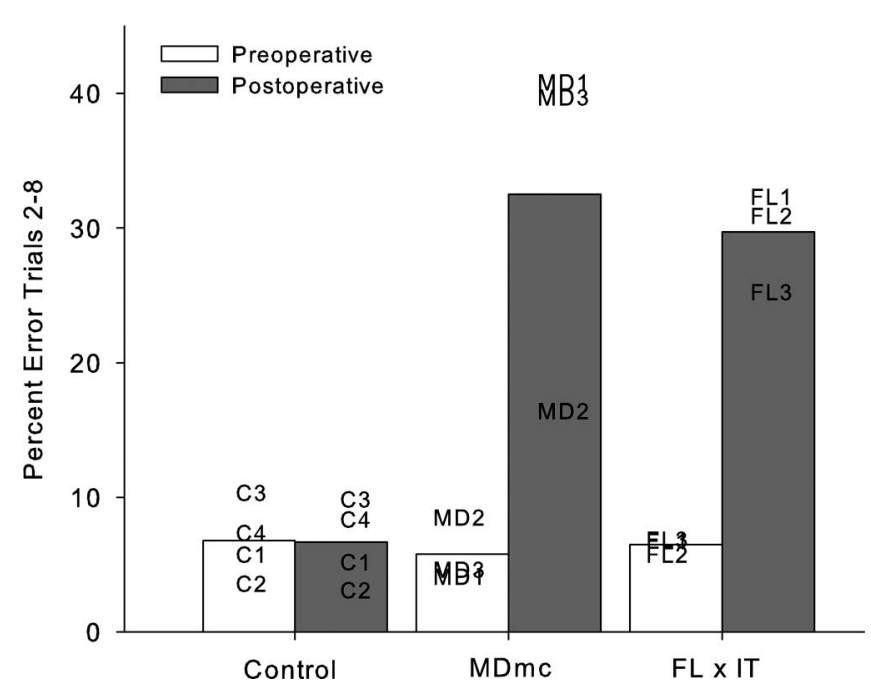

Figure 2. Scene learning. Mean percentage error on trials 2- 8 of lists of new scene learning during preoperative and postoperative performance tests for $\mathrm{MDmc}$, control, and full disconnection of frontal lobe from the inferotemporal cortex (FL by IT) (Browning et al., 2005) groups. Data presented are from individual animals.

Table 3. Percentage error in trial blocks 2-8 in scene learning

\begin{tabular}{lcc}
\hline Monkey & Preoperative & Postoperative \\
\hline C1 & 5.8 & 5.2 \\
C2 & 3.6 & 3.1 \\
C3 & 10.4 & 9.9 \\
C4 & 7.4 & 8.4 \\
Control mean & $\mathbf{6 . 8}$ & $\mathbf{6 . 6}$ \\
MD1 & 4.1 & 41.1 \\
MD2 & 8.6 & 16.5 \\
MD3 & 4.6 & 39.9 \\
MD neurotoxic mean $^{2}$ & $\mathbf{5 . 8}$ & 32.5 \\
S1 & 9.2 & 32.3 \\
S2 & & 27.1 \\
S3 $^{a}$ & 6.0 & 28.3 \\
MD ablation mean $^{a}$ & 15.2 & $\mathbf{2 9 . 2}$ \\
\hline
\end{tabular}

${ }^{a}$ Data presented by Gaffan and Parker (2000).

any perseveration tendency alone. Interestingly, Figure $4 b$ shows the types of response errors from a previously published group, frontal lobe (FL) by IT (Browning et al., 2005), and clearly indicates that this lesion group also performed in a similar manner, making more postoperative performance errors to both $1 \mathrm{C}$ and 1W trials.

\section{Strategy implementation}

The implementation of the strategy was measured at each stage as a mean ratio of the number of trials per reward made in each session from the final 10 sessions of the strategy implementation task during the double alternation performance test and is shown in Tables 1 and 2. It is clear that the monkeys had proficient and stable performance on the strategy implementation task during the preoperative performance test.

Postoperative implementation of the preoperatively acquired strategies is shown in Figure 5 and Tables 1 and 2. Neurotoxic lesions to the MDmc did not impair postoperative performance on the strategy implementation task when compared with the unoperated controls. This result was unexpected. A two (group, control vs MDmc) by two (testing phase, preoperative vs postoperative) repeated-measures ANOVA confirmed this. There was a main effect of testing phase $\left(F_{(1,5)}=11.57 ; p=0.02\right)$, but no

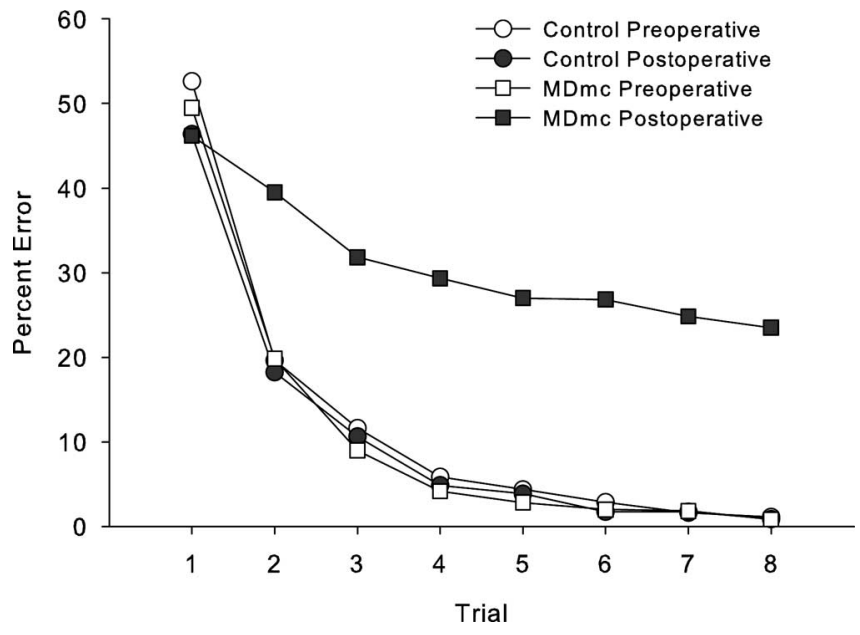

Figure 3. Scene learning. Mean learning curves for group neurotoxic magnocellular MDmc lesions and control monkeys during both preoperative and postoperative performance tests learning new sets of 20 scenes in eight repetition trials in a single session.

effect of group $\left(F_{(1,5)}=4.87 ; p=0.08\right)$, and, critically, no group by testing phase interaction $\left(F_{(1,5)}=1.29 ; p=0.31\right)$. Figure 4 and Tables 1 and 2 also include for comparison data from the FC by IT lesion group previously published by Gaffan et al. (2002), clearly indicating the substantial postoperative deficits produced by this lesion.

\section{Discussion}

The present study demonstrates for the first time and unexpectedly that neurotoxic lesions of the MDmc do not result in deficits at implementing a preoperatively acquired strategy task. That is, the MDmc lesion group performed at almost the same preoperative level of proficiency on strategy implementation. In contrast, neurotoxic MDmc lesions resulted in severe memory deficits during new scene learning, a macaque model for some aspects of episodic memory (namely object and place) (Figs. 2, 3). The current memory impairment in new learning is similar to previous results using the same task after MDmc ablations (Gaffan and Parker, 2000). In contrast, operated controls from a previously published study (Parker and Gaffan, 1997) that underwent similar surgical procedures to those mentioned above (see Materials and Methods, Surgery), but did not receive a lesion in the thalamus demonstrated no deficits during postoperative performance using the scene memory task.

The range of scene memory deficits across the three monkeys is interesting. The monkey who had the smallest memory impairments, MD2, also had the smallest lesion in the MDmc (see Materials and Methods, Histology). The other two monkeys with more extensive memory deficits had more extensive neuronal loss in the MDmc. These two monkeys also had additional thalamic damage, one with some unilateral damage in the right laterodorsal and anterodorsal thalamic nuclei and the other had some unilateral damage in the left anterior thalamus (see Materials and Methods, Histology). This unilateral thalamic damage is unlikely to have been the cause of the new learning impairments of these two monkeys as it has been shown that unilateral lesions in other structures do not cause deficits in new scene learning after entorhinal lesion (Charles et al., 2004), fornix transection (Gaffan and Parker, 1996), perirhinal lesion (Gaffan and Parker, 1996), ablation of frontal or inferotemporal cortex (Browning et al., 2005), or deficits in delayed matching-to-sample after unilateral MDmc ablations (Parker and Gaffan, 1998). Our MDmc 
lesions also produced additional damage to midline thalamic nuclei, including the paraventricular and central intermedialis nuclei lying between the MDmc in the two hemispheres of the thalamus. It has been established previously that these midline thalamic nuclei alone do not cause memory deficits as combined unilateral MDmc and midline thalamic nuclei lesions do not affect learning abilities of monkeys (Gaffan and Murray, 1990). Nevertheless the midline thalamic damage in combination with our bilateral MDmc lesions may have contributed to the severity of the cognitive deficits in our lesion group (Gaffan and Parker, 2000). Furthermore, previous anatomical tracing studies confirm strong connections between these midline thalamic nuclei and medial prefrontal cortex areas that are also interconnected with MDmc (Hsu and Price, 2007).

The methodology used in the current study is not directly comparable with previous studies using either the scene memory or strategy implementation tasks as these tasks were always run singly whereas the current study involved preoperative and postoperative performance tests of double sessions alternating between the two experiments. The addition of unoperated controls in the current study allowed for an assessment of this new methodology and clearly demonstrated that all of the monkeys were able to perform this double alternation testing after postsurgery recovery or an equivalent rest period. Thus the current results indicate two important conclusions. First, that the MDmc is a critical structure in scene memory. Second, the memory impairments caused by either neurotoxic or ablation lesion techniques are the result of damage to the MDmc and not caused by inadvertent damage to fibers of passage coursing through this region of the medial thalamus.

The current results from the strategy implementation task were unexpected. Gaffan et al. (2002) showed that the interactions between frontal and temporal cortices are important for strategies. On the view that the MDmc contributes to memory processes by disrupting normal functioning in PFC, impairments in strategy implementation would be expected after lesions of MDmc. The current results do not support this proposal, suggesting that the MDmc does not disrupt PFC functioning across all cognitive behavioral tasks that depend on an intact PFC. The dissociation between the two tasks in the current study indicates that the memory deficits resulting from MDmc lesions are not the result of a generalized dysfunction of PFC.

One possibility is that the MDmc does not contribute to the retrieval of preoperatively acquired information in general. The strategy implementation task does not require new learning or relearning per se, but rather simply implementation of a preoperatively acquired strategy based on retention of the same four sets of objects associated with the two different categories of rewards. Gaffan et al. (2002) proposed that during the strategy implementation task the PFC is selectively activated by the representation of the currently active strategy as well as being involved in retrieving the abstract rules whereas reciprocal temporal connections with posterior cortical areas could then activate the representations of the appropriate objects for that strategy. The lack of deficits after the lesions to the MDmc suggests that the interaction between the PFC and MDmc is not preventing the

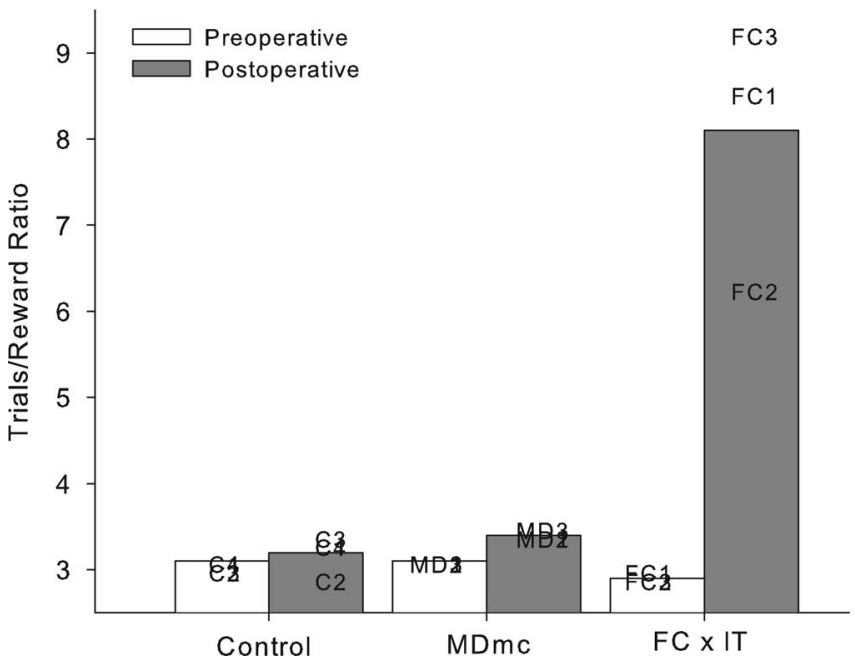

Figure 5. Strategy implementation. The mean trials/reward ratios for neurotoxic magnocelIular MDmc lesions, controls, and full disconnection of frontal lobe from the inferotemporal cortex (FC by IT) (Gaffan et al. (2002) monkeys during both preoperative and postoperative performance tests are shown. Data presented are from individual animals.

PFC from retrieving the abstract rules necessary to implement the strategy efficiently. A previously published study using a different cognitive task tentatively reached a similar conclusion. MDmc ablations did not impair retention of a list of 100 preoperatively overtrained object-reward associations (Gaffan and Parker, 2000). In contrast, a previous study has shown that MDmc ablations impair relearning of preoperatively acquired discriminations (Gaffan and Watkins, 1991). Thus, it appears that MDmc and its interactions with PFC are critical for relearning and new learning, but perhaps are not critical for retention. additional studies are clearly required to determine the extent of pure retention deficits after bilateral lesions of the MDmc on tasks that assess similar forms of associative memory, for example retention of preoperatively acquired memory for scenes verses postoperative learning of new scenes; our laboratory is currently investigating these hypotheses.

In contrast, the links between the MDmc and PFC are important in new learning. Crossed unilateral lesions of PFC and IT produce substantial impairments in postoperative new scene learning (Browning et al., 2005). This memory deficit is compa- 
rable with the results of the current study. In that same study, bilateral PFC lesions also caused substantial deficits in new scene learning.

It has been suggested that the mediodorsal thalamus like the PFC is important for the ability to shift from a preferred response to a nonpreferred one as changes occur in the rules of the task (Hunt and Aggleton, 1998). The results in the current study that analyzed the types of response errors $(1 \mathrm{C} / 1 \mathrm{~W})$ made indicated that the monkeys postoperatively were not simply perseverating with initial incorrect responses throughout each session, as they also made more errors when their initial response was correct (Fig. 4a). Furthermore, the types of response errors made by both the MDmc and crossed FC by IT lesion groups (Browning et al., 2005 ) were very similar (Fig. $4 b$ ). This type of responding suggests difficulties with acquiring the new stimulus-reward associations, rather than a problem with inhibiting responses or perseverative responding. Similar observations have been noted in previously reported studies of MD lesions in rats (Chudasama et al., 2001).

The scene memory task is a complex task assessing many aspects of rapid new learning including spatial, visual object and contextual information, stimulus-reward associations and perhaps even temporal order processing (Browning et al., 2005). It has been shown previously that the medial division of the mediodorsal thalamus does not have a role in spatial memory processing in rats (Aggleton and Brown, 1999; Mitchell and Dalrymple-Alford, 2005). However, impairments in temporal order processing for the presentations of objects are impaired after bilateral lesions to the medial division of the mediodorsal thalamus (Mitchell and Dalrymple-Alford, 2005), a task that is also sensitive to lesions of the medial frontal cortex in rats (Mitchell and Laiacona, 1998). Another possibility is that the MDmc may have a role in rapid new learning of reward value type associations, because of the prominent MDmc connections with the orbital prefrontal cortex, as this latter cortical region has been implicated in reward value type processing (Izquierdo et al., 2004). These possibilities are not mutually exclusive, as multiple areas of prefrontal cortex contribute to new scene learning, perhaps by different mechanisms (Wilson et al., 2007) (Baxter et al., 2007).

In conclusion, the current results provide greater insight into the function of MDmc in memory, and more clearly define the role of the MDmc in memory processes. It appears that the MDmc is important in new visual learning via its interactions with PFC, but once these new associations are formed within the cortex and the new learning is complete, the importance of the MDmc diminishes and does not contribute to memory retrieval. Lateral parts of the mediodorsal thalamic nucleus (i.e., the parvocellular and densocellular divisions) are also densely interconnected with the PFC. Future investigations determining the contribution of these lateral parts of the mediodorsal thalamus are now required to establish their roles in different memory processes.

\section{References}

Aggleton JP, Brown MW (1999) Episodic memory, amnesia, and the hippocampal-anterior thalamic axis. Behav Brain Sci 22:425-444, discussion $444-489$.

Aggleton JP, Mishkin M (1983) Memory impairments following restricted medial thalamic lesions in monkeys. Exp Brain Res 52:199-209.

Baxter MG, Gaffan D (2007) Asymmetry of attentional set in rhesus monkeys learning colour and shape discriminations. Q J Exp Psychol (Colchester) 60:1-8.

Baxter MG, Gaffan D, Kyriazis DA, Mitchell AS (2007) Orbital prefrontal cortex is required for object-in-place scene memory but not performance of a strategy implementation task. J Neurosci 27:11327-11333.

Browning PG, Easton A, Buckley MJ, Gaffan D (2005) The role of prefrontal cortex in object-in-place learning in monkeys. Eur J Neurosci 22:3281-3291.

Charles DP, Browning PG, Gaffan D (2004) Entorhinal cortex contributes to object-in-place scene memory. Eur J Neurosci 20:3157-3164.

Chudasama Y, Bussey TJ, Muir JL (2001) Effects of selective thalamic and prelimbic cortex lesions on two types of visual discrimination and reversal learning. Eur J Neurosci 14:1009-1020.

Gaffan D (1994) Scene-specific memory for objects: a model of episodic memory impairment in monkeys with fornix transection. J Cogn Neurosci 6:305-320.

Gaffan D, Murray EA (1990) Amygdalar interaction with the mediodorsal nucleus of the thalamus and the ventromedial prefrontal cortex in stimulus reward associative learning in the monkey. J Neurosci 10:3479-3493.

Gaffan D, Parker A (1996) Interaction of perirhinal cortex with the fornixfimbria: memory for objects and "object-in-place" memory. J Neurosci 16:5864-5869.

Gaffan D, Parker A (2000) Mediodorsal thalamic function in scene memory in rhesus monkeys. Brain 123:816-827.

Gaffan D, Watkins S (1991) Mediodorsal thalamic lesions impair long-term visual associative memory in macaques. Eur J Neurosci 3:615-620.

Gaffan D, Murray EA, Fabrethorpe M (1993) Interaction of the amygdala with the frontal-lobe in reward memory. Eur J Neurosci 5:968-975.

Gaffan D, Easton A, Parker A (2002) Interaction of inferior temporal cortex with frontal cortex and basal forebrain: double dissociation in strategy implementation and associative learning. J Neurosci 22:7288-7296.

Goldman-Rakic PS, Porrino LJ (1985) The primate mediodorsal (MD) nucleus and its projection to the frontal lobe. J Comp Neurol 242:535-560.

Hsu DT, Price JL (2007) Midline and intralaminar thalamic connections with the orbital and medial prefrontal networks in macaque monkeys. J Comp Neurol 504:89-111.

Hunt PR, Aggleton JP (1998) Neurotoxic lesions of the dorsomedial thalamus impair the acquisition but not the performance of delayed matching to place by rats: a deficit in shifting response rules. J Neurosci 18:10045-10052.

Ilinsky IA, Kultas-Ilinsky K (1987) Sagittal cytoarchitectonic maps of the Macaca mulatta thalamus with a revised nomenclature of the motorrelated nuclei validated by observations on their connectivity. J Comp Neurol 262:331-364.

Izquierdo A, Suda RK, Murray EA (2004) Bilateral orbital prefrontal cortex lesions in rhesus monkeys disrupt choices guided by both reward value and reward contingency. J Neurosci 24:7540-7548.

Mitchell AS, Dalrymple-Alford JC (2005) Dissociable memory effects after medial thalamus lesions in the rat. Eur J Neurosci 22:973-985.

Mitchell JB, Laiacona J (1998) The medial frontal cortex and temporal memory: tests using spontaneous exploratory behaviour in the rat. Behav Brain Res 97:107-113.

Parker A, Gaffan D (1997) Mamillary body lesions in monkeys impair object-in-place memory: functional unity of the fornix-mamillary system. J Cogn Neurosci 9:512-521.

Parker A, Gaffan D (1998) Interaction of frontal and perirhinal cortices in visual object recognition memory in monkeys. Eur J Neurosci 10:3044-3057.

Parker A, Eacott MJ, Gaffan D (1997) The recognition memory deficit caused by mediodorsal thalamic lesion in non-human primates: a comparison with rhinal cortex lesion. Eur J Neurosci 9:2423-2431.

Russchen FT, Amaral DG, Price JL (1987) The afferent input to the magnocellular division of the mediodorsal thalamic nucleus in the monkey, Macaca fascicularis. J Comp Neurol 256:175-210.

Ungerleider LG, Gaffan D, Pelak VS (1989) Projections from inferior temporal cortex to prefrontal cortex via the uncinate fascicle in rhesus monkeys. Exp Brain Res 76:473-484.

Victor M, Adams RD, Collins GH (1989) The Wernicke-Korsakoff syndrome, Ed 2. Philadelphia: F. A. Davis.

Wilson CRE, Gaffan D, Mitchell AS, Baxter MG (2007) Neurotoxic lesions of ventrolateral prefrontal cortex impair object-in-place scene memory. Eur J Neurosci 25:2514-2522.

Zola-Morgan S, Squire LR (1985) Amnesia in monkeys after lesions of the mediodorsal nucleus of the thalamus. Ann Neurol 17:558-564. 